\title{
AVALIAÇÃO DA REPRODUTIBILIDADE INTEROBSERVADORES DE UMA NOVA ESCALA PARA ORIENTAÇÃO DA CONDUTA TERAPÊUTICA NAS METÁSTASES VERTEBRAIS - ESCORE SINS (SPINE INSTABILITY NEOPLASTIC SCORE)
}

\author{
ASSESSMENT OF INTEROBSERVER REPRODUCIBILITY OF A NEW SCALE FOR \\ MANAGEMENT GUIDANCE IN SPINAL METASTASIS - SINS SCORE
}

\author{
EVALUACIÓN DE LA REPRODUCIBILIDAD INTEROBSERVADOR DE UNA NUEVA ESCALA \\ PARA LA ORIENTACIÓN DE LA CONDUCTA TERAPÊUTICA DE LAS METÁSTASIS \\ VERTEBRALES - PUNTUACIÓN SINS
}

Hugo José Sousa Sales da Silva1, Marcelo Italo Risso Neto², Raphael de Rezende Pratali³, Guilherme Rebecchi Zuiani², Paulo Tadeu Maia Cavali², Ivan Guidolin Veiga ${ }^{2}$, Wagner Pasoualini ${ }^{2}$, Maurício Antonelli Lehoczki ${ }^{4}$, Alexander Junoueira Rossato ${ }^{4}$, Élcio Landim ${ }^{5}$

\section{RESUMO}

Objetivo: Avaliar o impacto na conduta e quantificar a reprodutibilidade interobservador do escore SINS. Além disso, determinar sua aplicabilidade em nosso meio. Métodos: Compilou-se uma apresentação com 20 casos de lesões secundárias da coluna, que foi analisada por 10 observadores. Estes definiram a estabilidade de cada lesão e, após a apresentação do sistema SINS, os mesmos casos foram apresentados aos mesmos observadores para que novamente determinassem a estabilidade da lesão. Os dados colhidos foram analisados por meio do cálculo do Kappa de Fleiss e da correlação intraclasse. Resultados: Obtivemos concordância moderada interobservador com o uso do escore SINS. Conclusões: 0 sistema é aplicável em nosso meio. Houve mudança de opinião quanto à estabilidade da lesão após a apresentação do escore aos observadores.

Descritores: Classificação; Coluna vertebral; Metástase neoplásica; Estabilização; Neoplasias da coluna vertebral.

\begin{abstract}
Objective: To evaluate the impact on the management and quantify the inter-observer reproducibility of SINS Score. Also, determine its applicability to our milieu. Methods: A presentation of 20 cases of secondary spine lesions was compiled, which was examined by 10 observers. They defined the stability of each injury and, after the presentation of SINS Score, the same cases were presented to the same observers and again they were asked to determine the stability of the lesion. The collected data were analyzed by the Fleiss' Kappa Calculation and Intra-class Correlation. Results: Inter-observer moderate agreement was obtained by using SINS classification. Conclusions: The classification is applicable to our milieu. There was a change of opinion about the stability of the lesions after the presentation of the classification to the observers.
\end{abstract}

Keywords: Classification; Spine; Neoplasm metastasis; Stabilization; Spinal neoplasms.

\section{RESUMEN}

Objetivo: Evaluar el impacto en la conducta y cuantificar la reproducibilidad interobservador de la escala SINS. Además de eso, determinar la aplicabilidad de la clasificación en nuestro medio. Métodos: Se compiló una presentación de 20 casos de lesiones secundarias de la columna vertebral que fue examinada por 10 obsenvadores. Estos definieron la estabilidad de cada lesión y después de la presentación de la puntuación SINS, los mismos casos fueron presentados a los mismos observadores para que de nuevo determinasen la estabilidad de la lesión. Los datos obtenidos fueron analizados mediante el cálculo de Kappa de Fleiss y de la correlación intraclasse. Resultados: Obtuvimos concordancia moderada interobservador con el uso de la puntuación SINS. Conclusiones: La puntuación es aplicable en nuestro medio. Hubo un cambio de opinión en cuanto a la estabilidad de la lesión después de la presentación de la puntuación a los observadores.

Descriptores: Clasificación; Columna vertebral; Metástasis de la neoplasia; Estabilización; Neoplasias de la columna vertebral.

\section{INTRODUÇÃO}

Doença metastática na coluna vertebral consiste em um problema de saúde pública, cuja incidência é crescente, parte pelo aumento da incidência de doenças neoplásicas na população geral, parte pelo avanço em técnicas mais modernas no diagnóstico e tratamento da doença primária, permitindo a esses pacientes expectativa de vida mais longa. Assim, com o aumento na prevalência de pacientes oncológicos com doença metastática na coluna, é importante o desenvolvimento de planos de tratamento, com abordagem multidisciplinar entre oncologistas, radioterapeutas e

\footnotetext{
1. Médico Estagiário da Disciplina de Cirurgia da Coluna do Departamento de Ortopedia eTraumatologia da Universidade Estadual de Campinas (Unicamp) - Campinas, SP, Brasil. 2. Médico Assistente da Disciplina de Cirurgia da Coluna do Departamento de Ortopedia eTraumatologia da Universidade Estadual de Campinas (Unicamp), Campinas, SP, Brasil. 3. Médico Colaborador da Disciplina de Cirurgia da Coluna do Departamento de Ortopedia eTraumatologia da Universidade Estadual de Campinas (Unicamp), Campinas, SP, Brasil. 4. Médico do Grupo de Escoliose da Associação de Assistência à Criança Deficiente (AACD) - São Paulo, SP, Brasil.

5. Professor e Chefe da Disciplina de Cirurgia da Coluna do Departamento de Ortopedia eTraumatologia da Universidade Estadual de Campinas (Unicamp), Campinas, SP, Brasil.
}

Trabalho realizado junto ao Departamento de Ortopedia e Traumatologia da Faculdade de Ciências Médicas da Universidade Estadual de Campinas - Unicamp. SP, Brasil. 
cirurgiões de coluna, visando a determinação do melhor tratamento a ser empregado. Muitos sistemas de classificação têm sido desenvolvidos com intuito de guiar o tratamento das doenças neoplásicas na coluna vertebral.

Em 1986, Harrington ${ }^{1}$ publicou um escore para doenças metastáticas da coluna dividida em cinco categorias, dependendo da extensão do comprometimento neurológico ou da destruição óssea. Baseado nessa classificação orientou o tratamento das lesões em tratamento oncológico ou cirúrgico.

Em 1989 e 1990, Tokuhashi et al.,3 propuseram um escore pré-operatório para definir o prognóstico de sobrevida dos pacientes com metástases na coluna para selecionar as opções de tratamento. Esse escore foi baseado em seis parâmetros, incluindo condições gerais (performance status), metástases ósseas extra-espinhais, número de metástases vertebrais, metástases em órgãos internos, histologia do tumor primário e quadro neurológico. Analisando esses parâmetros, criou-se uma pontuação que orientou a uma das modalidades de tratamento, incluindo tratamento conservador, cirurgia paliativa ou cirurgia excisional.

Após a descrição de abordagem mais agressiva, conhecida como Espondilectomia Total em bloc ${ }^{4}$, outros conceitos oncológicos passaram a ser levados em consideração na obtenção de controle local bem sucedido das lesões na coluna. Em 2001, Tomita et al. ${ }^{5}$ propuseram um novo sistema de escore baseado em prognóstico das doenças metastáticas da coluna vertebral, propondo um guideline para tratamento dessas lesões. Foram considerados três parâmetros, incluindo histologia do tumor primário, metástases viscerais e metástases ósseas, cada um desses parâmetros recebendo pontos para atingir um escore prognóstico dividido em quatro grupos, cada um recebendo uma estratégia cirúrgica: suporte básico, cirurgia paliativa, cirurgia excisional intralesional ou marginal e cirurgia com margem ampla.

Em novembro de 2010, Gasbarrini et al. ${ }^{6}$ apresentaram a experiência um sistema algoritmo para o tratamento de metástases na coluna vertebral. Analisando características clínicas do paciente, status neurológico, características do tumor primário, como sensibilidade à quimioterapia, radioterapia ou terapia hormonal, o sistema orienta entre tratamento paliativo, cirurgia descompressiva e/ou estabilização, ou cirurgia excisional.

Em pacientes com doença neoplásica metastática na coluna vertebral não existe um consenso na decisão de quais pacientes devem ser submetidos a tratamento cirúrgico, sendo que muitas metástases vertebrais respondem favoravelmente a métodos não cirúrgicos como radioterapia, quimioterapia ou tratamento hormonal. O quadro neurológico do paciente é apenas um fator que influencia na decisão para cirurgia, além do estado geral do paciente, prognóstico, histologia do tumor primário e estabilidade. O conceito de instabilidade vertebral é crítico no processo de decisão para tratamento cirúrgico.

Recentemente, em 2010, um grupo de especialistas em oncologia da coluna vertebral publicou um sistema de escore visando guiar a identificação de instabilidade vertebral devido à doença neoplásica (Spinal Instability Neoplastic Score- SINS) ${ }^{7}$. Baseado em um processo de incorporação de medicina baseada em evidência com o que há de melhor disponível na literatura e opinião de experts, foi desenvolvido um sistema de escore considerando-se seis parâmetros: localização da lesão, dor, característica radiográfica da lesão óssea, alinhamento vertebral radiográfico, colapso do corpo vertebral e envolvimento dos elementos posteriores da vértebra. Atribuindo-se pontos a cada parâmetro, pode-se classificar a estabilidade vertebral: pontuação entre 0 e 6 denota "estabilidade", entre 7 e 12 "instabilidade indeterminada (iminente)" e entre 13 e 18 denota "instabilidade". Os autores sugerem que pacientes com pontuação entre 7 e 18 sejam encaminhados para avaliação com especialistas em cirurgia de coluna.

Com base na metodologia cuidadosa do grupo que publicou o escore SINS, acreditamos que este sistema de escore seja de aplicação segura. No entanto necessita de validação na literatura para popularizar seu uso. Reprodutibilidade interobservador significa que os observadores diferentes concordarão em opinião quanto à classificação analisando um mesmo caso. Uma boa classificação deve ter adequada e documentada reprodutibilidade interobservador ${ }^{8}$.

Este trabalho objetiva avaliar a aplicabilidade do sistema de escore SINS em nosso meio, através de dados clínicos e radiográficos de pacientes já submetidos a tratamento. Também buscamos avaliar qual teria sido seu impacto na orientação do tratamento desses pacientes, além de quantificar a reprodutibilidade interobservador do escore SINS

\section{MÉTODOS}

Recolhemos dados retrospectivamente de prontuários pacientes adultos com lesões neoplásicas secundárias do ambulatório da FCM- UNICAMP. Organizamos uma apresentação de Power Point com 20 casos de lesões neoplásicas da coluna com dados clínicos e exames de imagem de modo que foi possível a determinação de pontuação pelo SINS. Não incluímos casos com lesões contíguas, já que os autores do SINS desencorajaram a aplicação do escore para tais casos. Os casos foram apresentados a nove ortopedistas especialistas em coluna e um neurocirurgião para determinação de conduta antes de ser apresentado sistema de escore SINS. Num segundo momento, apresentamos o sistema de escore SINS e os mesmos casos foram então apresentados aos mesmos observadores para determinação da estabilidade. Documentamos a concordância ou não da conduta antes e posterior ao uso do SINS. Suas respostas foram armazenadas para cálculo da reprodutibilidade interobservador. Foram calculados os coeficientes Kappa de Fleiss ${ }^{9}$ para avaliar a concordância na classificação de instabilidade e tratamento entre os observadores (interobservadores) na conduta espontânea e com uso da escala SINS. Para avaliar a reprodutibilidade interobseravadores da escala SINS foi calculada a correlação intraclasse com o respectivo intervalo com 95\% de confiança ${ }^{9}$. Para estes cálculos utilizamos os softwares Excel 2003, R 2.14.0 e SAS 9.1.

\section{RESULTADOS}

Os resultados estão dispostos na Tabela 1 e na Tabela 2:

Tabela 1. Confiança interobservador para a determinação da instabilidade e do tratamento entre os observadores ${ }^{9}$.

\begin{tabular}{c|c|c|c}
\hline & Reprodutibilidade & \multicolumn{2}{|c}{ IC (95\%) } \\
\hline & Interobservador-Kappa & inferior & superior \\
\hline $\begin{array}{c}\text { Estabilidade-Conduta } \\
\text { espontânea }\end{array}$ & 0,334 & 0,238 & 0,430 \\
\hline $\begin{array}{c}\text { Tratamento- } \\
\text { Conduta espontânea }\end{array}$ & 0,248 & 0,173 & 0,323 \\
\hline Estabilidade- SINS & 0,503 & 0,429 & 0,577 \\
\hline Tratamento - SINS & 0,265 & 0,184 & 0,346 \\
\hline
\end{tabular}

IC: Intervalo de confiança.

Tabela 2. Correlação intraclasse para avaliação da reprodutibilidade da escala interobservadores ${ }^{9}$.

\begin{tabular}{c|c|c|c}
\hline Variável & Correlação Intraclasse & \multicolumn{2}{|c}{ IC (95\%) } \\
\hline & & inferior & superior \\
\hline Escore SINS & 0,854 & 0,761 & 0,928 \\
\hline
\end{tabular}

IC: Intervalo de confiança.

\section{DISCUSSÃO}

Toumey ${ }^{10}$ estudou as metástases à coluna em seus pacientes e observou a dor lombar crônica como sendo o principal dado de suspeição do diagnóstico. Como modalidades terapêuticas não incluíam tratamento cirúrgico, mas indicava tratamento com colete de Taylor para os pacientes com dor de ritmo mecânico, sendo esse seu parâmetro indicativo de instabilidade, apesar de não ter utilizado tal termo.

Young e Funk ${ }^{11}$ avaliaram as metástases na coluna lombar em uma correlação entre os achados patológicos e radiográficos e 
concluem que as lesões blásticas são observadas mais precocemente nas radiografias e que as lesões líticas somente seriam observadas ao acometerem a cortical óssea, quando podem ser observadas inclusive fraturas.

Harrington ${ }^{12}$ descreveu que os sintomas relacionados à metástase espinhal podem estar relacionados à instabilidade. Ele classificou os pacientes com metástase para a coluna em cinco categorias em que segrega o acometimento neurológico do acometimento ósseo e associa a instabilidade ao colapso vertebral. Define instabilidade conforme as teorias e Denis ${ }^{13}$ e White e Panjabi ${ }^{14}$.

Galasko et a $/{ }^{15}$ publicaram uma revisão sobre conceitos atuais em instabilidade espinhal secundária a câncer metastático e em momento algum no texto é encontrada uma forma de definição para a instabilidade. Avaliam como um problema o não-encaminhamento dos pacientes com metástase na coluna ao cirurgião de coluna e verificam as causas da não-referência como sendo o desconhecimento do problema pelos médicos oncologistas e radioterapeutas; e como culpa dos cirurgiões de coluna a formação inadequada dos ortopedistas gerais que não sabem referenciar os pacientes ao especialista em tempo correto

Com o objetivo de propor um sistema que determinasse objetivamente a presença ou ausência de instabilidade espinhal por neoplasia, em 2010 um grupo internacional de pesquisadores interessados em problemas de coluna, autodenominados SOSG publicou seu trabalho. Foi montado um sistema de escores para definir se determinada lesão é estável, instável ou se há instabilidade iminente. $O$ problema da não-referência de pacientes ao especialista em coluna também tem uma solução proposta pelo grupo, que seria encaminhar ao cirurgião de coluna todos os pacientes cujos escores pelo seu sistema (SINS) fossem de lesão com instabilidade iminente ou mesmo instáveis; ou seja, devem ser encaminhados ao cirurgião de coluna para avaliação todos os pacientes com lesões não-estáveis. Na conclusão de seu estudo os autores mesmo afirmam que após validação e testes de reprodutibilidade adicionais o seu sistema poderá ser bastante útil aos médicos assistentes de pacientes com metástases espinhais ${ }^{7}$.

Obviamente, a estabilidade da lesão é tão somente um dos aspectos a serem avaliados na decisão da conduta terapêutica dos casos, mas necessita realmente de maior atenção dos pesquisadores e melhor definição de termos. Vemos o sistema SINS como um marco importantíssimo no estudo das neoplasias espinhais, já que é utilizada uma única ferramenta de avaliação para toda a coluna; possui aplicabilidade a todos os médicos assistentes, inclusive determinando a tão indefinida, mas também sempre citada instabilidade espinhal.

A Tabela 1 e a Tabela 2 mostram reprodutibilidade adequada do sistema de escore. Sendo a melhor reprodutibilidade conseguida interobservadores com o uso do escore SINS na determinação da estabilidade, já a reprodutibilidade no tratamento com e sem a escala foram bastante similares. O mesmo observador classificou a lesão de uma forma antes do conhecimento do sistema de escore SINS. Após a apresentação do escore SINS, a mesma lesão avaliada pelo mesmo observador no mesmo dia foi classificada de outra forma quanto à estabilidade. Aguardamos mais dados da literatura para saber se o uso do sistema de escore determinará ou não modificações nas condutas. De qualquer forma, em nosso estudo, as condutas foram modificadas pelo conhecimento do sistema de escore SINS pelos observadores.

\section{CONCLUSÃO}

O sistema SINS é perfeitamente aplicável em nosso meio. Houve divergência entre as avaliações quanto à estabilidade das lesões antes e após a aplicação do SINS, o que talvez venha a influenciar em uma mudança de opinião destes especialistas ao aplicarem o SINS. Entretanto, a conduta terapêutica não foi modificada por este novo escore. O escore possui moderada reprodutibilidade interobservador.

\section{REFERÊNCIAS}

1. Harrington KD. Metastatic disease of the spine. J Bone Joint Surg Am. 1986:68(7):1110-5

2. Tokuhashi $Y$, Kawano H, Ohsaka S, Matsuzaki H, Toriyama S. [A scoring system for preoperative evaluation of the prognosis of metastatic spine tumor (a preliminary report)]. Nihon Seikeigeka Gakkai Zasshi. 1989:63(5):482-9.

3. Tokuhashi Y, Matsuzaki H, Toriyama S, Kawano H, Ohsaka S. Scoring system for the preoperative evaluation of metastatic spine tumor prognosis. Spine (Phila Pa 1976). 1990:15(11):1110-3

4. Tomita K, Kawahara N, Baba H, Tsuchiya H, Fujita T, Toribatake Y. Total en bloc spondylectomy. A new surgical technique for primary malignant vertebral tumors. Spine (Phila Pa 1976). 1997;22(3):324-33

5. Tomita K, Kawahara N, Kobayashi T, Yoshida A, Murakami H, Akamaru T. Surgical strategy for spinal metastases. Spine (Phila Pa 1976). 2001;26(3):298-306.

6. Gasbarrini A, Li H, Cappuccio M, Mirabile L, Paderni S, Terzi S, et al. Efficacy evaluation of a new treatment algorithm for spinal metastases. Spine (Phila Pa 1976). 2010:35(15):1466-70.

7. Fisher CG, DiPaola CP, Ryken TC, Bilsky MH, Shaffrey Cl, Berven SH, et al. A novel classification system for spinal instability in neoplastic disease: an evidence-based approach and expert

consensus from the Spine Oncology Study Group. Spine (Phila Pa 1976). 2010;35(22):E1221-9.

8. Chan P, Boriani S, Fourney DR, Biagini R, Dekutoski MB, Fehlings MG, et al. An assessment of the reliability of the Enneking and Weinstein-Boriani-Biagini classifications for staging of primary spinal tumors by the Spine Oncology Study Group. Spine (Phila Pa 1976). 2009:34(4):384-91

9. Fleiss JL. The design and analysis of clinical experiments. New York:Wiley; 1986

10. Toumey JW. Metastatic malignancy to the spine. J Bone Joint Surg. 1943;25(2):292-395.

11. Young JM, Funk FJ Jr. Incidence of tumor metastasis to the lumbar spine; a comparative study of roentgenographic changes and gross lesions. J Bone Joint Surg Am. 1953; 35(1):55-64.

12. Harrington KD. Current concepts review. Metastaic disease to the spine. J Bone Join Surg Am. 1986;68(7):1110- 5.

13. Denis F. Spinal instability as defined by the three-column spine concept in acute spinal trauma. Clin Orthop Relat Res. 1984;(189):65-76

14. White AA 3rd, Panjabi MM. Clinical biomecanic of the spine. Philadelphia: JB Lippincott; 1978.

15. Galasko CS, Norris HE, Crank S. Spinal Instability Secondary to Metastatic Câncer: Current Concepts Review. J Bone Joint Surg Am. 2000 Apr;82(4):570-76. 